male with severe TBI.

A 40-year-old male patient was brought to Emergency Department (ED) in an unconscious state with alleged history of road traffic accident. Patient was immediately intubated in the ED and his postresuscitation Glasgow Coma Scale (GCS) score was E1VetM5. Pupils bilaterally were of normal size, sluggishly reacting to light. Systemic examination did not reveal any associated orthopedic or abdominal solid organ injury. Focused abdominal sonogram for trauma (FAST) was also negative. Urgent noncontrast computed tomography (NCCT) head revealed right fronto-tempero-parietal acute subdural hematoma (SDH) with mass effect and significant midline shift to the left side [Figure 1]. He was immediately taken up for emergency surgery. Large right fronto-tempero-parietal decompressive craniectomy was done, SDH was evacuated and lax duraplasty was done using pericranial graft. Three hours after surgery when the patient did not reverse from the effect of anesthesia, we got a CT head repeated which revealed a large right cerebellar hemorrhage with mass effect over fourth ventricle with upstream hydrocephalus [Figure 2]. Coagulation parameters were normal, both pre- and postoperatively. Patient had been operated in supine position on a horse-shoe headrest. No three-point fixator was used for headholding during the operative procedure. There was no history of trauma to head during shifting. Urgent external ventricular drainage (EVD) was done at the bedside to relieve hydrocephalus. However, patient became brain-dead before definitive surgery could be offered and expired a day later.

\title{
Cerebellar hemorrhage
} following supratentorial decompressive craniectomy for severe traumatic brain \section{injury}

Sir,

Postoperative hemorrhage is a well-known complication following intracranial surgery, and usually results from inadequate hemostasis. Remote site hemorrhage i.e., hemorrhage occurring at a distant site away from the site of craniotomy, is relatively rare and may occasionally cause significant morbidity or even death. ${ }^{[1-3]}$ Only a few cases of infratentorial hemorrhage following supratentorial surgery were reported,,$^{[4-6]}$ and those following surgery for traumatic brain injury (TBI), are a rarity. We report a case of infratentorial hematoma following ipsilateral decompressive hemicraniectomy for traumatic acute subdural hematoms in a 40 yeary-old
Remote site intracranial hemorrhage is an extremely rare complication of intracranial surgery. ${ }^{[1,2]}$ The complication has been reported following tumor excision ${ }^{[2]}$ and also following evacuation of chronic subdural hematoma. ${ }^{[3]}$

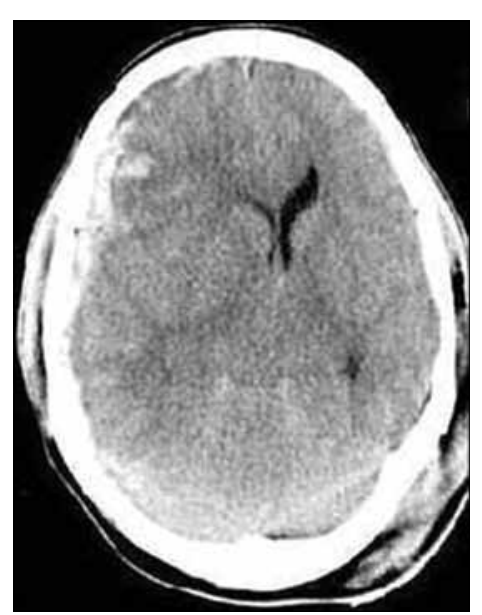

Figure 1: Right fronto-parietal acute subdural hematoma with mass effect and midline shift to left side 


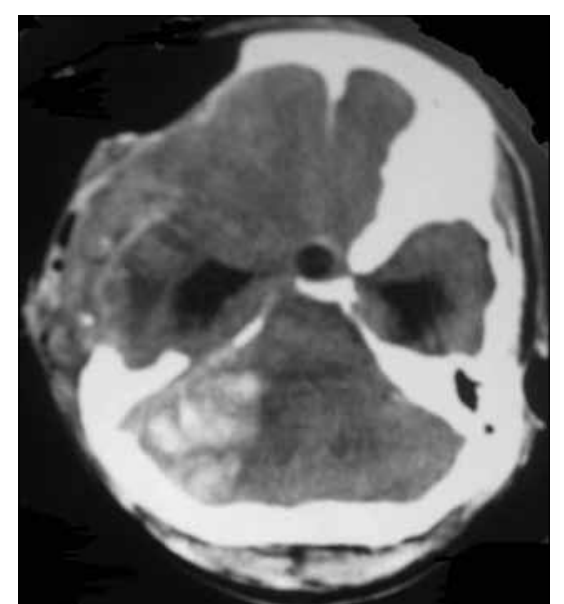

Figure 2: Development of right cerebellar hematoma following supratentorial fronto-tempero-parietal decompressive craniectomy

There are also isolated reports of infratentorial hemorrhage following supratentorial surgery and vice-versa. ${ }^{[4-6]}$ The pathophysiology of this entity is poorly understood though various hypotheses have been put forward. Initially it was thought that transient hypertensive peaks during recovery from anesthesia was the causative factor. But recent studies have refuted this theory as only very few patients had documented high blood pressure readings perioperatively, thus suggesting probably surgery itself may be a contributing factor in inducing coagulation abnormalities. It has also been hypothesized that the tissue thromboplastin released from the injured brain tissue in severe TBI leads to a local consumptive coagulopathy. The loss of substantial cerebrospinal fluid (CSF) volume during surgery appears to play a central role in the pathophysiological development of remote site hemorrhage. ${ }^{[1]}$ Suction of the CSF may cause intracranial hypotension. Further reduction of intracranial pressure leads to an increased transluminal venous pressure with subsequent rupture of vessels. ${ }^{[1]}$ Substantial loss of CSF leads to sagging of the cerebellum away from the tentorium and thus stretching of the cerebellar veins with an increase in the transmural pressure or it may be due to jugular vein compression by the transverse process of atlas in extended neck position.$^{[6]}$ Cerebellar "sag" as a result of CSF hypovolemia, causing transient occlusion of superior bridging veins within the posterior fossa and consequent hemorrhagic venous infarction, has been proposed to be the most likely pathophysiological cause of remote cerebellar hemorrhage. The loss of CSF volume within the cisterns and ventricles is likely to draw interstitial fluid into the cerebellar compartment leading to increased venous pressure and venous hemorrhage.

To conclude, remote site hemorrhage is a potentially fatal complication after supratentorial neurosurgical procedures, especially those involving the opening of CSF cisterns or the ventricular system. There is not a single preoperative or intraoperative factor that can reliably predict the occurrence of remote site hemorrhages, and the etiology of this entity remains unclear. The differential diagnosis of declining level of consciousness after supratentorial surgery must include remote site hemorrhage and CT scan of the head should be done to exclude this complication. The most important keys to minimize the hazardous sequelae are to be aware of this potential complication, a high index of suspicion, a prompt diagnosis and to provide emergent treatment in all cases.

\section{Sachin A. Borkar, G. Lakshmi Prasad, Deepak Agrawal, B. S. Sharma}

Department of Neurosurgery, Jai Prakash Narayan Apex Trauma Center, All India Institute of Medical Sciences, New Delhi, India. E-mail:drdeepak@gmail.com

PMID: 19934581

\section{References}

DOI: $10.4103 / 0028-3886.57800$

1. Landeiro JA, Flores MS, Lapenta MA, Galdino AC, Lázaro BC. Remote hemorrhage from the site of craniotomy. Arq Neuropsiquiatr 2004;62:832-4.

2. Kalfas IH, Little JR. Postoperative hemorrhage: A survey of 4992 intracranial procedures. Neurosurgery 1988;23:343-7.

3. Dinc C, Iplikcioglu AC, Bikmaz K, Navruz Y. Intracerebral hemorrhage occurring at remote site following evacuation of chronic subdural hematoma. Acta Neurochir (Wien) 2008;150:497-9.

4. Tondon A, Mahapatra AK. Supratentorial intracerebral hemorrhage following infratentorial surgery. J Clin Neurosci. 2004;11:762-5.

5. Gurjar H, Dwarakanath S, Sharma BS, Chandra S. Cerebellar hemorrhage following endoscopic third ventricular colloid cyst decompression. Neurol India 2008;56:218-20.

6. Friedman JA, Piepgras DG, Duke DA, McClelland RL, Bechtle PS, Maher CO, et al. Remote cerebellar hemorrhage after supratentorial surgery. Neurosurgery 2001;49:1327-40.

Accepted on 27-08-2009 